\title{
Balancing mitochondrial biogenesis and mitophagy to maintain energy metabolism homeostasis
}

\author{
K Palikaras ${ }^{1,2}$, E Lionaki $^{1}$ and N Tavernarakis ${ }^{*, 1,3}$
}

Cell Death and Differentiation (2015) 22, 1399-1401; doi:10.1038/cdd.2015.86

Mitochondria, the main energy hub of the cell, are highly dynamic organelles, playing essential roles in fundamental cellular processes. Mitochondrial function impinges on several signalling pathways modulating cellular metabolism, cell survival and healthspan. Accordingly, impairment of mitochondria has been associated with numerous pathological conditions and ageing. Maintenance of cellular and organismal homeostasis thus hinges on fine-tuning mitochondrial quality control. Mitochondrial biogenesis and mitochondrial selective autophagy (mitophagy), two opposing cellular pathways, coordinately regulate mitochondrial content to sustain energy metabolism, in response to cellular metabolic state, stress and other intracellular or environmental signals. It is not surprising, therefore, that disequilibrium or imbalance between mitochondrial proliferation and degradation processes underlies the onset and progressive unfolding of several pathological conditions in humans, including neurodegenerative diseases, myopathies and other age-associated disorders.

Mitochondrial biogenesis is a complex and multistep cellular process, which involves mtDNA transcription and translation, translation of transcripts derived from nucleus, recruitment of newly synthesized proteins and lipids, import and assembly of mitochondrial and nuclear products in the expanding mitochondrial network. Spatiotemporal control of mitochondrial biogenesis is mediated by numerous transcription factors in response to diverse stimuli, including both intracellular signals and environmental stimuli (nutrient availability, growth factors and hormones, toxins, temperature and oxygen fluctuations, among others). The master regulator of mitochondrial energy metabolism is the peroxisome proliferator-activated receptor gamma co-activator 1-alpha (PGC-1a), the best-studied member of the peroxisome proliferator activated receptor family of transcription co-activators, which orchestrates the activity of several transcription factors involved in mitochondrial biogenesis and function. ${ }^{1}$ These include the nuclear respiratory factors (NRF1 and NRF2), the estrogen-related receptors (ERR- $\alpha,-\beta$ and $-\gamma$ ) and the nuclear factor erythroid 2-related factor 2 (NRF2/NFE2L2) that are part of a complex transcriptional network that regulates mitochondrial biogenesis and energy metabolism. ${ }^{1,2}$

Alongside their essential roles in cell and animal physiology, mitochondria are also the major source of potentially hazardous reactive oxygen species as by-products of respiration. Thus, eukaryotic cells have evolved a wide arsenal of quality control mechanisms to preserve mitochondrial homeostasis and prevent cellular damage and eventual death. Mitophagy, a selective type of autophagy, is triggered upon accumulation of damaged or superfluous mitochondria. Dysfunctional mitochondria are targeted and engulfed by double-membrane vesicles known as autophagosomes and are transferred for degradation in lysosomes. Cells induce mitophagy to regulate the size and quality of their mitochondrial network in response to energy demands.

Excessive build-up of mitochondria is a common feature of various cell types, in several pathological conditions and during ageing. ${ }^{3-6}$ The reason underlying this phenomenon remained elusive. We found that age-related mitophagy decline causes progressive accumulation of mitochondria in the nematode Caenorhabditis elegans. Inhibition of either general autophagy or specifically mitophagy increases mitochondrial mass, recapitulating the effects of ageing in young individuals. Mitophagy is markedly induced by low insulin/IGF-1 signalling and contributes to lifespan extension under these conditions. Moreover, mitophagy promotes longevity upon mild attenuation of mitochondrial function or by dietary restriction. In addition to its pro-longevity effects, mitophagy confers resistance to various stressors, including starvation, genotoxic, mitochondrial and oxidative stress. ${ }^{3}$

Maintenance of mitochondrial homeostasis requires both clearance of damaged and generation of fresh, functional mitochondria. Emerging evidence indicates that coordination between mitochondrial biogenesis and mitophagy is essential for proper mitochondrial homeostasis. Several molecules have been implicated in the fine-tuning of coordination. Sirtuins modulate energy metabolism and longevity through their function as histone deacylases. For example, upon fasting SIRT1 deacetylates PGC-1a to induce mitochondrial gene-expression and biogenesis in skeletal muscles. ${ }^{7}$ In addition, SIRT1 activity stimulates autophagy, whereas SIRT1 deficiency causes accumulation of aberrant mitochondria. Converging evidence suggests that SIRT1 targets components of the autophagic machinery to exert its effects on autophagy. ${ }^{8}$ However, the exact mechanism remains elusive. The metabolic sensor AMP-activated protein kinase (AMPK) that becomes activated upon high energy demands has also

\footnotetext{
${ }^{1}$ Institute of Molecular Biology and Biotechnology, Foundation for Research and Technology-Hellas, Heraklion, Crete, Greece; ${ }^{2}$ Department of Biology, University of Crete, Heraklion, Crete, Greece and ${ }^{3}$ Department of Basic Sciences, Faculty of Medicine, University of Crete, Heraklion, Crete, Greece

${ }^{*}$ Corresponding author: N Tavernarakis, Institute of Molecular Biology and Biotechnology, Foundation for Research and Technology-Hellas, Nikolaou Plastira 100, Vassilika Vouton, Heraklion 70013, Crete, Greece. Tel: +30 2810 391069; Fax: +30 2810 391067; E-mail: tavernarakis@imbb.forth.gr
} 


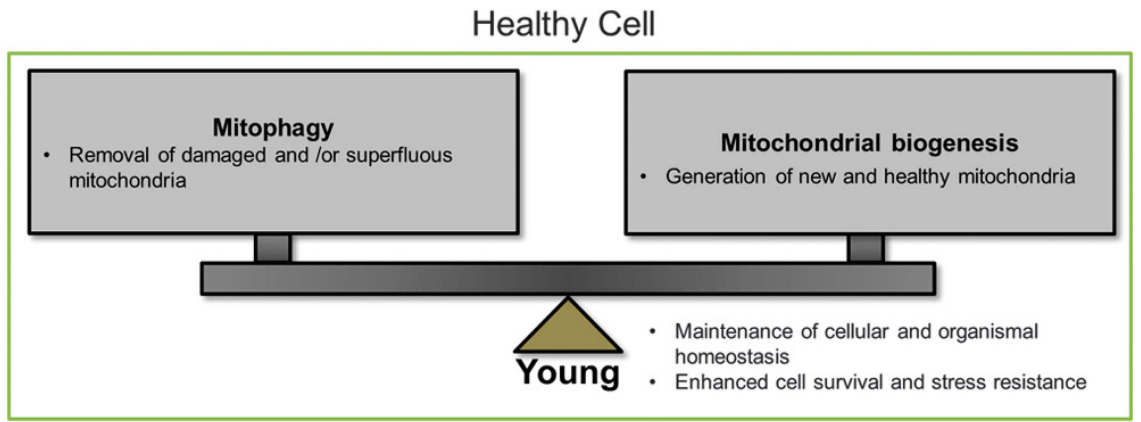

Aged Cell

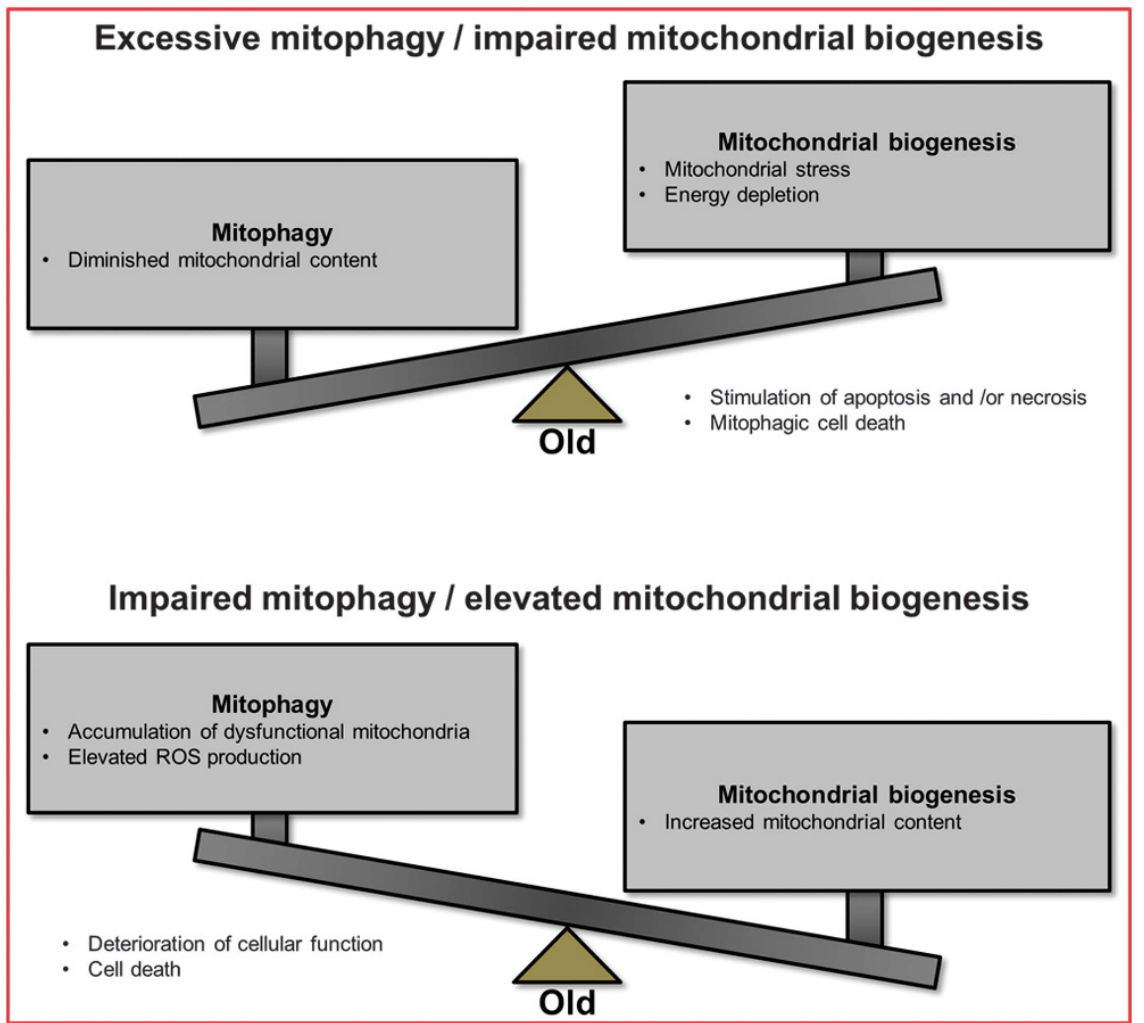

Figure 1 Metabolic homeostasis by coordination of mitochondrial biogenesis and mitophagy. The close entanglement of mitochondrial biogenesis and mitophagy ensures the removal of damaged and/or superfluous mitochondria, as well as the balanced generation of new organelles. Maintenance of energy metabolism homeostasis is achieved by coordinating these two processes, which promotes cell survival and stress resistance. Disequilibrium between mitochondrial biogenesis and selective autophagy causes deterioration of cellular function and cell death

been implicated in the regulation of mitochondrial biogenesis through PGC-1 $a^{9}$ Loss of AMPK and its downstream target ULK1, a kinase that inhibits autophagy, causes aberrant accumulation of the p62-adaptor-protein and impairment of mitophagy during starvation. ${ }^{10}$ Furthermore, p53, the master regulator of genotoxic stress response, is involved in the maintenance of energy homeostasis. p53 functions both in the nucleus and mitochondria and influences mitochondrial physiology in a transcription-dependent and -independent manner. These effects are mediated mainly by induction of mitochondrial biogenesis and modulation of autophagy..$^{11}$ In conclusion, Parkin, the E3 ubiquitin ligase that triggers tagging and clearance of damaged mitochondria through mitophagy, also interfaces with mitochondrial biogenesis. This link is established through association of Parkin with TFAM, mtDNA and the PGC-1a transcriptional inhibitor PARIS. ${ }^{12-14}$

Our work reveals a key regulatory mechanism that couples mitochondrial biogenesis with mitophagy to regulate energy metabolism in C. elegans. The nematode NRF2/NFE2L2 homolog, SKN-1, is a nodal element of the mechanism. Similarly to its mammalian counterpart, SKN-1 is activated by oxidative stress in response to mitochondrial dysfunction and drives the expression of several mitochondrial biogenesis genes. SKN-1 transcriptional activity is essential for mitohormesis-mediated longevity and maintenance of mitochondrial homeostasis. ${ }^{15,16}$ Notably, the mitophagy mediator gene dct-1 is among SKN-1 targets. DCT-1, the homolog of the mammalian BNIP3 and BNIP3L/NIX, is an integral outer mitochondrial membrane protein, which facilitates mitophagy 
by interacting with the autophagosomal protein LGG-1 (the C. elegans LC3 homolog).

NRF2/NFE2L2 is tethered to mitochondria through association with Keap1 and PGAM5 proteins. ${ }^{17} \mathrm{SKN}-1$ was also found to interact with the outer mitochondrial protein PGAM-5. ${ }^{18}$ Calcium signalling modulates mitochondrial biogenesis through the calcium/calmodulin-dependent protein kinase II (CaMKII), p38 mitogen activated protein kinase (MAPK) and PGC-1a. ${ }^{19,20}$ Although, no clear PGC-1a homolog is present in the nematode genome, we found that SKN-1 activity is modulated by cytoplasmic calcium levels via the CaMKII homolog, UNC-43, upon mitochondrial dysfunction.

Our findings, in their totality, indicate that mitochondrial biogenesis and mitophagy are co-regulated by SKN-1. Mitophagy is a pivotal component of this mechanism, which augments stress resistance and promotes longevity. We suggest that SKN-1 associates with mitochondrial components and acts as biosensor of mitochondrial integrity and function. The DCT-1 mitophagy mediator is a key converging point of intracellular and environmental signals that impinge on mitochondria removal. Accumulation of damaged or superfluous mitochondria imposes oxidative stress, initiating a bipartite retrograde response that is mediated by $\mathrm{SKN}-1$ and involves the coordinated induction of both mitochondrial biogenesis and mitophagy genes. Uncoupling of these two opposing processes during ageing contributes to accretion of dysfunctional mitochondria and consequently, deterioration of cellular function (Figure 1). Although our understanding of the complex regulatory network underlying mitochondrial homeostasis is still rather limited, further dissection of relevant molecular mechanisms will provide important insight into how cellular energy metabolism can be manipulated to benefit organismal physiology.

\section{Conflict of Interest}

The authors declare no conflict of interest.

1. Dominy JE, Puigserver P. Cold Spring Harb Perspect Biol 2013; 5: a015008.

2. Yun J, Finkel T. Cell Metab 2014; 19: 757-766.

3. Palikaras K, Lionaki E, Tavernarakis N. Nature 2015.

4. Artal-Sanz M, Tavernarakis N. Nature 2009; 461: 793-797.

5. Malpass K. Nat Rev Neurol 2013; 9: 417.

6. Wredenberg A et al. Proc Natl Acad Sci USA 2002; 99: 15066-15071.

7. Gerhart-Hines Z et al. EMBO J 2007; 26: 1913-1923.

8. Lee IH et al. Proc Natl Acad Sci USA 2008; 105: 3374-3379.

9. Jager $S$ et al. Proc Natl Acad Sci USA 2007; 104: 12017-12022.

10. Egan DF et al. Science 2011; 331: 456-461.

11. Sahin E, DePinho RA. Nat Rev Mol Cell Biol 2012; 13: 397-404

12. Kuroda $Y$ et al. Hum Mol Genet 2006; 15: 883-895.

13. Rothfuss $O$ et al. Hum Mol Genet 2009; 18: 3832-3850.

14. Shin JH et al. Cell 2011; 144: 689-702.

15. Schmeisser S et al. Aging Cell 2013; 12: 508-517.

16. Staab TA et al. PLoS Genet 2014; 10.

17. Lo SC, Hannink M. Exp Cell Res 2008; 314: 1789-1803.

18. Paek J et al. Cell Metab 2012; 16: 526-537.

19. Wright DC et al. J Biol Chem 2007; 282: 18793-18799.

20. Wu H et al. Science 2002; 296: 349-352. 